\title{
On the Detection of Frequency-Spectra-Based Chipless RFID Using UWB Impulsed Interrogation
}

\author{
Prasanna Kalansuriya, Student Member, IEEE, Nemai Chandra Karmakar, Senior Member, IEEE, and \\ Emanuele Viterbo, Fellow, IEEE
}

\begin{abstract}
A novel approach is presented to accurately estimate the resonant features of a multipatch backscatter-based chipless radio frequency identification (RFID) tag. An ultra-wideband impulse radar (UWB-IR)-based reader interrogates the chipless tag with a UWB pulse, and the received backscatter is analyzed in the time domain. The key components constituting the backscattered signal, the structural mode, and the antenna mode are identified, and their spectral content are analyzed. Analysis shows that the antenna mode backscatter contains the information carrying signal while the structural mode backscatter contains no information about the tag. A semi-analytical model is developed to explain the behavior of the signal backscattered from the chipless RFID tag. Simulation and semi-analytical results are validated by experimental measurements obtained in an anechoic chamber environment using a 4-b multipatch chipless RFID tag. The new method does not rely on calibration tags for operation and has a greater degree of freedom in the orientation of tags with respect to a reader. A novel method, selective spectral interrogation (SSI), that uses a set of interrogation pulses to extract information bits stored in the spectral signature of the chipless tag is also introduced.
\end{abstract}

Index Terms-Backscatter, chipless radio frequency identification (RFID), ultra-wideband impulse radar (UWB-IR), time domain.

\section{INTRODUCTION}

$\mathbf{R}$ ADIO FREQUENCY identification (RFID) has automated the process of item tracking and data entry involved in a multitude of applications in a diverse set of industries ranging from logistics and inventory management systems to food and agriculture. The fully automatic long-range operation has been the key feature that differentiates RFID from other automatic identification and tagging technologies, such as one-dimensional (1-D) and two-dimensional (2-D) optical barcodes. The advances in semiconductor technology and tag manufacturing technology has enabled mass production of RFID tags at very low costs, as low as $7-15$ cents. However, the cost of individual RFID tags are still significant to present

Manuscript received July 10, 2012; revised September 08, 2012; accepted September 13, 2012. Date of publication November 15, 2012; date of current version December 13, 2012. This work was supported in part by the Australian Research Council's Linkage Project under Grant LP0991435 and Express Promotions Australia Pty Ltd. This paper is an expanded paper from the IEEE MTT-S International Microwave Symposium, Montreal, QC, Canada, June $17-22,2012$.

The authors are with the Department of Electrical and Computer Systems Engineering, Monash University, Clayton, VIC, 3800 Australia (e-mail: prasanna.kalansuriya@monash.edu; nemai.karmakar@monash.edu; emanuele.viterbo@monash.edu).

Color versions of one or more of the figures in this paper are available online at http://ieeexplore.ieee.org.

Digital Object Identifier 10.1109/TMTT.2012.2222920 barriers to entry into applications involving very large-scale item tagging, such as library management systems where optical barcodes are predominately used.

Chipless RFID [1] has recently gained much interest as a solution to reduce the costs in mass deployment of RFID technology. The conventional passive RFID consists of an antenna for harvesting energy from the signals transmitted by an RFID reader and an integrated electronic circuit or chip that assists in the wireless communication process. The chip also contains an electronic product code (EPC) that is transmitted back to the reader by the chip through digital remodulation of the reader signal. On the other hand, a chipless RFID tag contains no electronic circuitry and is void of any capacity for intelligent signal processing, which makes it less expensive and easily mass produced at a unit cost comparable to optical barcodes. It is therefore, essentially the RF counterpart of an ordinary optical barcode.

Research on chipless RFID tags can be broadly classified into two main categories: time-domain reflectometry (TDR)-based chipless RFID and frequency-signature-based chipless RFID. In TDR-based tags, the RFID reader transmits an ultra-wideband (UWB) RF interrogation pulse and listens to the reflections or echoes coming back from the tag [2]-[7]. By varying the structural properties of the tag, the time of arrival of these echoes can be controlled, providing a method for passive data storage in the tag. In frequency-signature-based chipless RFID tags [8]-[10], the frequency spectrum of the interrogation signal sent by the RFID reader is transformed by the tag to represent data bits. Most of the research on these frequency signature or frequency-spectra-based tags use planar microwave circuits to realize these transformations in the amplitude or phase spectra of the backscattered signals.

Frequency-spectra-based RFID systems and TDR-based RFID systems have their own advantages and disadvantages. The operation of TDR-based chipless RFID systems inherently requires the use of a UWB impulse radar (UWB-IR)-based reader architecture [6], [7]. This is because the interrogation of the tag is done by using an extremely short-duration (lasting only a few hundred picoseconds) broadband RF pulse. This IR-based reader architecture provides a low-cost means for reader design, as opposed to the frequency-modulated continuous-wave (FMCW)-based readers [8], [11] used for reading frequency-spectra-based chipless RFID tags, which require expensive wideband voltage-controlled oscillators (VCOs). From the work reported in literature, it is generally observed that more information can be stored in frequency-spectra-based chipless tags [12], [13] than in TDR-based tags. However, TDR-based chipless tags have a greater degree of freedom in 
positioning the tag with respect to the reader [14] and can be detected successfully up to several meters [6]. Also, they do not require the use of calibration tags or calibration ground planes that need to be placed at the tag location in order to obtain reference measurements as in [8] and [15].

The use of UWB-IR-based interrogation for frequencyspectra-based chipless RFID has not received much attention [15]-[17]. By using UWB-IR-based interrogation of frequency-spectra-based chipless RFID and analyzing the backscatter in the time domain, it is possible to combine the benefits of both classes of chipless RFID systems. This will give rise to the development of chipless RFID operating at longer ranges with a higher data capacity and that are less constrained on positioning, orientation, and calibration requirements. The authors of [15] use the singularity expansion method to characterize the response of a chipless RFID tag to an RF impulse. The response is expressed using a set of poles and residues that are in turn used in the representation of the information encoded in the chipless RFID tag. The proposed technique is experimentally verified. However, reading the tag involves in a three-step measurement procedure: 1) a first calibration measurement in an empty room; 2) a second calibration measurement with a ground plane placed at the location of the tag; and 3) the tag measurement. Therefore, it is not suitable for an application where the distance to the tag varies or where the environment is highly dynamic. In [16], UWB pulsed interrogation of a spiral resonator-based chipless RFID is considered where a meandering transmission line is used to isolate and show that the resonance information of the spirals is contained in the antenna mode of the backscatter. The research detailed in [17] analyzes the backscatter from a multipatch-based chipless RFID tag that is read using a UWB-IR-based reader. In both [16] and [17], the proposed methods do not require a calibration tag to aid the detection process and solely relies on the backscattered signal to estimate the resonance information of the tag. However, the methods are not validated using experimental results and are based only on electromagnetic simulations.

In this paper, we explore the use of UWB impulse-based interrogation for remotely estimating the resonant features of a backscatter-based multipatch chipless RFID tag as in [17]. We extend and validate the work reported in [17] through further analysis and by using experimental results. The proposed system uses a single antenna configuration for interrogating the tag and receiving its backscatter. The single-antenna arrangement provides a wider degree of freedom in orienting the tag with respect to the reader. It is shown that the resonant information of the chipless tag is contained only in the antenna mode of the backscatter using electromagnetic simulation results. A mathematical model based on semi-analytical approximations is also derived to explain the behavior of the backscatter and to show how the individual components of the system contribute to the formation of the total received signal. The simulation and analytical results are also validated by measurement results obtained through experiments conducted in an anechoic chamber environment. The operation of the proposed system does not require calibration ground planes or calibration tags, and the new method gives acceptable performance under different tag orientations and positions with respect to the reader location. Finally, a novel detection method called selective spectral interrogation

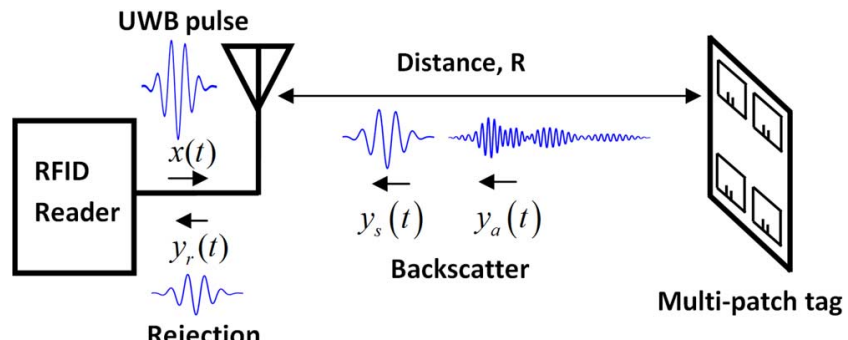

Fig. 1. Multipatch chipless RFID tag and reader system.

(SSI) is introduced in order to accurately detect the information stored in the chipless tag amidst false spectral peaks caused by noise and clutter. The method uses a set of interrogation pulses, where each pulse is designed to maximize a unique resonance of the tag. It is shown that, by employing a peak detection algorithm together with these interrogation pulses, it is possible to accurately extract the information contained in the frequency spectrum of the chipless tag.

The remainder of this paper is organized as follows. Section II describes the chipless RFID system model considered in the paper. Section III presents the electromagnetic simulation results and analyzes the key components of the signal received at the RFID reader. The derivation of a semi-analytical approximation that explains the behavior of the tag backscatter is presented in Section IV. Experimental validation of the chipless RFID system and the performance of the chipless RFID tag through measurement results are discussed in Section V. Section VI details the representation of digital data in the tag frequency spectrum and provides a detection algorithm to extract the encoded information bits from the chipless tag. Finally, conclusions are drawn in Section VII.

\section{SySTEM MODEL}

Here, the system model of the chipless RFID system is discussed. An overview of the backscattering process is also detailed, and the major components of the backscattered signal are introduced.

Fig. 1 illustrates the RFID system considered in this analysis. The RFID reader consists of a single antenna serving as both a transmitter and a receiver. The tag consists of $N$ inset-fed microstrip patch antennas each resonating at a distinct frequency $f_{i}$, where $i=1, \ldots, N$. The signal $x(t)$ is the UWB impulse used for interrogating the chipless RFID tag. The total received signal $y(t)$ consists of three components

$$
y(t)=y_{r}(t)+y_{s}(t)+y_{a}(t) .
$$

The largest and the first received component $y_{r}(t)$ is the rejection of the transmit pulse $x(t)$ due to the return loss profile of the antenna. The transients of $y_{r}(t)$ gradually decay down to zero. At this moment in time, the reader antenna has fully transmitted $x(t)$ and is receptive to any backscatter coming from the tag. The second component received $y_{s}(t)$ is the structural mode of the backscatter. This is followed by the antenna mode of the backscatter $y_{a}(t)$, which is the weakest and the last component to be received [18], [19]. Let $S_{11}(f)$ be the return loss profile of the antenna. From the definition of the return loss, the rejected 
portion $y_{r}(t)$ of the pulse input into the antenna can be written as

$$
y_{r}(t)=\mathcal{F}^{-1}\left\{S_{11}(f) X(f)\right\}
$$

where $\mathcal{F}^{-1}(\cdot)$ denotes the inverse Fourier transform. Throughout the paper, we will use lower case letters to denote time-domain signals and the upper case letters to denote the respective frequency-domain signal, i.e., $X(f)=\mathcal{F}\{x(t)\}$. Due to the presence of a tag in front of the transmit/receive antenna, the original return loss of the antenna, $S_{11}(f)$, slightly changes. The return loss of the antenna is affected by the backscatter incident on the antenna and is considered to be electromagnetically loaded by the chipless tag. Let $S_{11}^{\text {Loaded }}(f)$ be the modified or affected return loss of the antenna. Using $S_{11}^{\text {Loaded }}(f),(1)$ can be rewritten as

$$
y(t)=\mathcal{F}^{-1}\left\{S_{11}^{\text {Loaded }}(f) X(f)\right\} .
$$

From (1)-(3), we can write an expression for $y_{s}(t)$ and $y_{a}(t)$, which introduces the electromagnetic loading in the reader antenna, as follows:

$$
y_{s}(t)+y_{a}(t)=\mathcal{F}^{-1}\left\{\left[S_{11}^{\text {Loaded }}(f)-S_{11}(f)\right] X(f)\right\} .
$$

Equation (4) is particularly useful in calculating the time-domain backscatter from the frequency-domain antenna return loss measurements taken using a vector network analyzer (VNA). Section V will discuss in detail the experimental validation of the system.

\section{System VALidation Using ElectromagnetiC SIMULATION}

Here, we present the operation of the chipless RFID system using electromagnetic simulation results. Here, the frequency spectra of the key components making up the backscatter are analyzed. In order to obtain a backscattered signal close to realistic conditions, the entire system shown in Fig. 1 was constructed in CST Microwave Studio [20] as a three-dimensional (3-D) model, and full-wave electromagnetic simulation was performed.

\section{A. UWB Transmission}

The UWB pulse used in the simulation is a modulated Gaussian pulse having a 20-dB bandwidth of $6 \mathrm{GHz}$ spanning from 2 to $8 \mathrm{GHz}$. The transmitted interrogation pulse can be expressed as

$$
x(t)=A_{0} \cos \left(2 \pi f_{c} t\right) \exp \left(-\frac{(t-\mu)^{2}}{2 \sigma^{2}}\right)
$$

where $\mu=0.6 \mathrm{~ns}$ and $\sigma=0.114 \mathrm{~ns}$ are the parameters defining the shape of the envelope Gaussian pulse, and $A_{0}=1$ and $f_{c}=5 \mathrm{GHz}$ are, respectively, the amplitude and the frequency of the sinusoidal carrier signal. Fig. 2(a) shows the shape of the transmitted pulse, and Fig. 2(b) shows its frequency spectrum. The pulse is transmitted using a coplanar waveguide (CPW)-fed circular disc loaded monopole antenna that operates from 2 to $7.3 \mathrm{GHz}$.

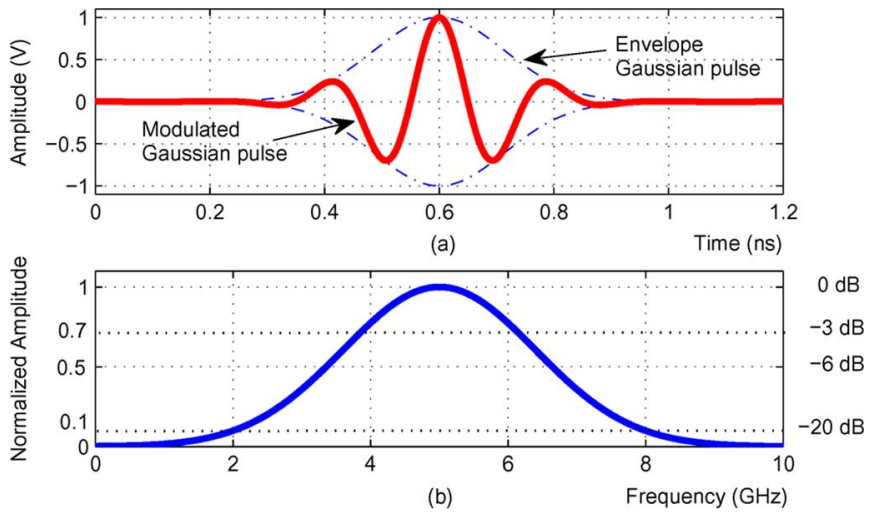

Fig. 2. (a) UWB interrogation pulse shape. (b) Frequency spectrum of pulse.
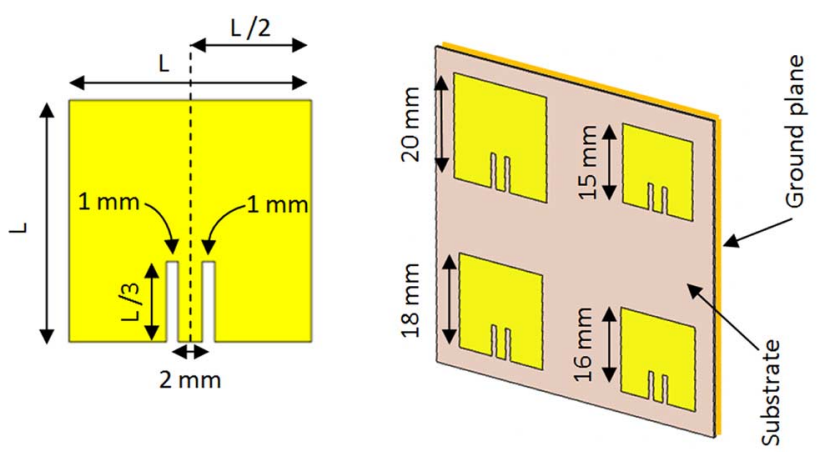

(a)

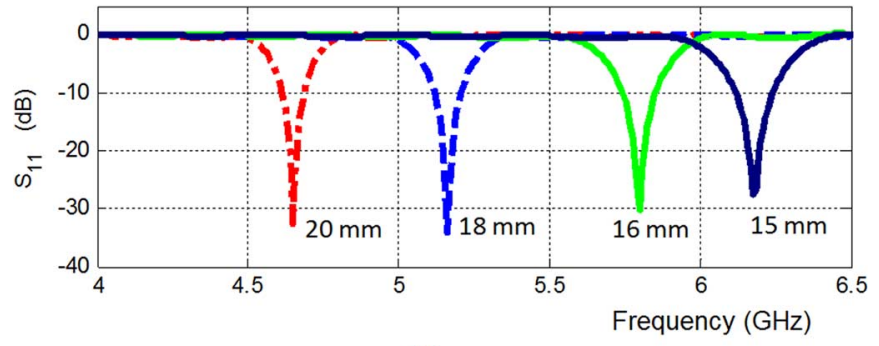

(b)

Fig. 3. (a) Chipless tag consisting of four inset-fed patch antennas resonating at four distinct frequencies. The dimension $L$ of a patch determines its resonant frequency. The design used a substrate of Taconic TLX-8 with $\epsilon=2.55$ and thickness $0.5 \mathrm{~mm}$. (b) $S_{11}$ characteristics of each patch antenna.

\section{B. Tag Design and Operation}

Fig. 3 shows the tag used in this paper. The tag considered in this work is similar to that in [9] and consists of an array of four inset-fed microstrip patch antennas. Each individual patch antenna is a half-wavelength resonator that resonates at a distinct frequency. By varying the dimensions of the patches, the tag can be engineered to have a unique spectral signature or a transfer function characterized by a set of resonances. This signature can be used to store information. The tag shown in Fig. 3 consists of four square patch antennas, having widths of 20,18 , 16 , and $15 \mathrm{~mm}$, which resonate at 4.64, 5.16, 5.8, and $6.2 \mathrm{GHz}$, respectively. The patches also have directive radiation patterns, where the 20-, 18-, 16-, and 15-mm patches respectively show $8,8.2,8.7$, and $8.2 \mathrm{dBi}$ of directivity. In this work, we focus on the amplitude spectrum as opposed to the phase spectrum as in [9]. When the transmitted UWB pulse interacts with the 


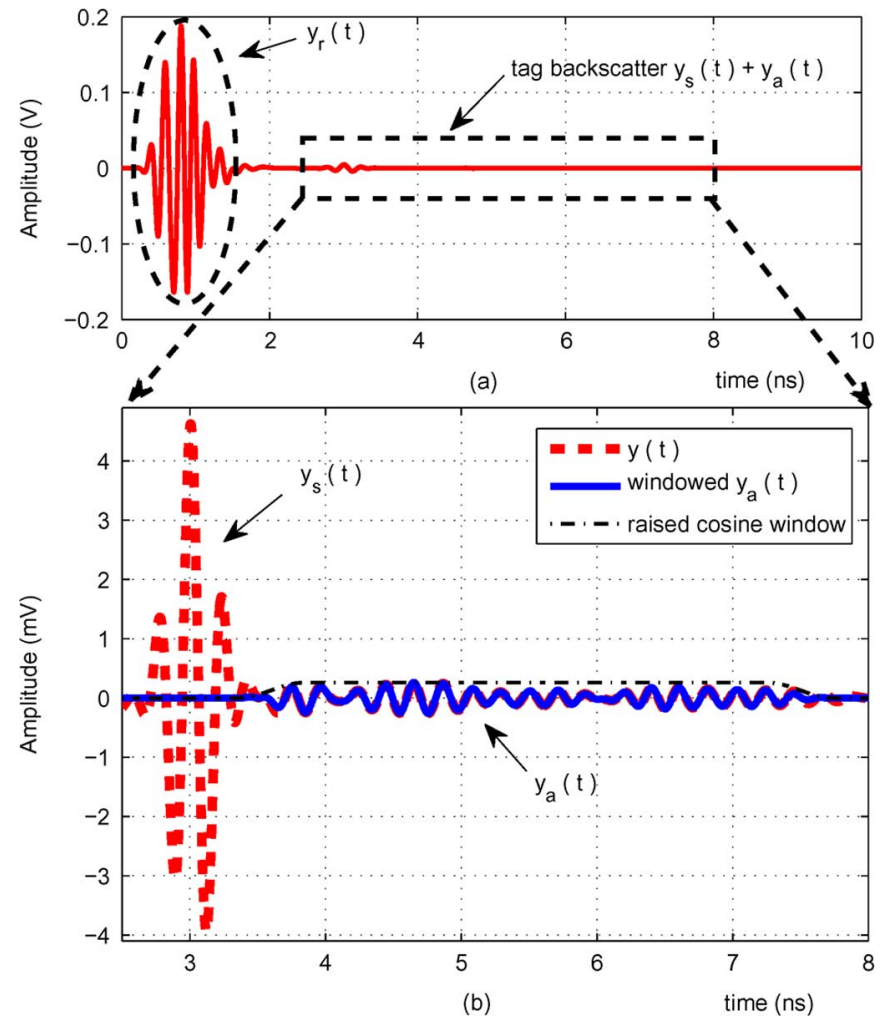

Fig. 4. (a) Complete received signal $y(t)$ at the antenna (b) Enlarged portion of $y(t)$ showing $y_{s}(t)$ and $y_{a}(t)$.

tag, part of it is harnessed by the individual patch antennas constituting the tag and another part of it is immediately reflected. The initial reflection $y_{s}(t)$ is caused by the size and shape of the metallic structure of the tag irrespective of the resonant properties of the tag patch antennas and is termed the structural mode of the backscatter [18]. Following this initial backscatter is a secondary backscatter, the antenna mode $y_{a}(t)$, which is made up of the signals captured by the individual patch antennas at their respective resonant frequencies. The strength of this reradiated signal is determined by the loading condition of each patch antenna. In this work, we have considered an open-circuit loading condition to maximize the antenna mode backscatter.

\section{Analysis of Tag Backscatter}

Fig. 4 shows the complete received signal $y(t)$ when the tag is placed $30 \mathrm{~cm}$ away from the reader antenna. Once the initial rejection $y_{r}(t)$ has faded away, it is clearly observed that the reader antenna picks up the backscatter from the tag after a propagation delay of $2.55 \mathrm{~ns}$. The backscatter consists of a larger component followed by transients. We hypothesize that the larger component is the structural mode $y_{s}(t)$, and the transients make up the $y_{a}(t)$. However, since there is no transmission line between the patch antennas and their open circuited termination as in [14], it is difficult to clearly define where $y_{s}(t)$ ends and $y_{a}(t)$ starts. Fig. 5 shows the spectral content of the windowed structural mode and windowed antenna mode obtained using the discrete Fourier transform (DFT). The DFT was computed using the fast Fourier transform (FFT) algorithm. A raised cosine window was used to approximately window out $y_{s}(t)$ and $y_{a}(t)$. It is clear that $y_{s}(t)$, which is the larger and first

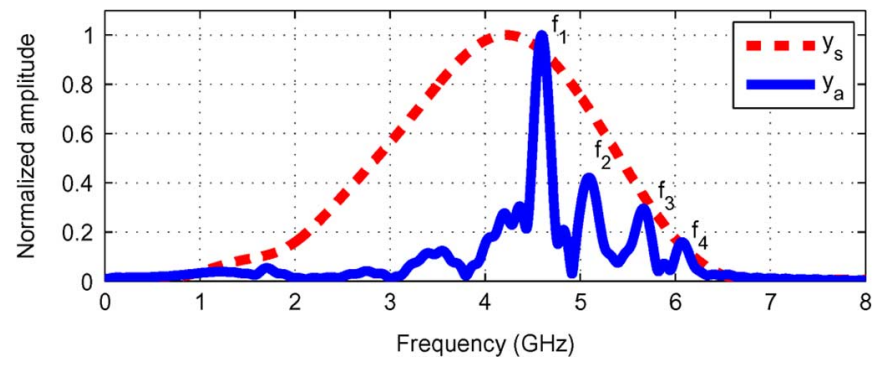

Fig. 5. Normalized amplitude spectrum of $y_{s}(t)$ and $y_{a}(t)$. The amplitude spectrum of $y_{s}(t)$ has a Gaussian shape similar to the input pulse whereas the spectrum of $y_{a}(t)$ shows four spectral peaks corresponding to the four resonant frequencies of the patch antennas in the tag.

portion of the backscatter, has a Gaussian amplitude spectrum similar to the spectrum of the transmitted UWB pulse [refer to Fig. 2(b)] and does not contain any information of the resonant frequencies of the tag. On the other hand, the spectral content of the windowed $y_{a}(t)$ clearly reveals the four resonant frequencies $\left(f_{1}=4.6, f_{2}=5.1, f_{3}=5.7\right.$, and $\left.f_{4}=6.1 \mathrm{GHz}\right)$ of the individual patch antennas. Therefore, it is clear that the transients $\left(y_{a}(t)\right)$ following the initial strong backscatter $\left(y_{s}(t)\right)$ holds the information required to estimate the resonant frequencies of the patch antennas in the chipless tag. It is also observed that the height of the peaks corresponding to the resonances closely follow a contour of a Gaussian amplitude spectrum. This is partly because the amplitude spectra of the transmitted pulse is Gaussian as seen in Fig. 2(b). The antenna mode simply consists of a filtered version of the transmitted signal where signals corresponding to the resonances will only be present.

\section{Semi-Analytical Approximation for tag BACKSCATTER}

The full-wave electromagnetic simulation performed using CST Microwave Studio provides a very accurate and realistic result. However, it does not provide insight into how the different components of the system contribute to produce the final result. Also, if a small change is made to the system, in order to understand its effect, the whole system needs to be resimulated. This is not desirable since full-wave electromagnetic simulation using CST consumes a large amount of computational resources and time.

Here, we present the derivation of a simplified mathematical model for describing the backscatter from the tag. The model approximates the entities (e.g., reader antenna, wireless channel, and patch antennas of the tag) constituting the total system as linear time-invariant (LTI) subsystems [21] that can each be fully described using a specific transfer function. The model developed here requires the knowledge of the return loss profiles of the reader antennas and the patch antennas of the tag, which are usually known beforehand or can be easily obtained through measurement or simulation. Therefore, it is not a complete analytical solution but a semi-analytical approximation. It is presented to validate and augment the intuitive discussion presented in Section III. Furthermore, it is capable of providing a reasonably accurate solution very quickly with reasonably low computational requirements. In this model, we assume that the tag and the reader antenna are perfectly polarization matched. 


\section{A. Structural Mode of Tag Backscatter}

In this approximation, we model the structural mode of the backscatter as a signal that simply gets reflected off of a reflective surface, where the spectral content of the reflected signal is not altered. Here, the structural mode backscatter $y_{s}(t)$ is simply an attenuated version of the signal that is emitted by the reader antenna into the free space, which is in turn picked up by the same antenna after a propagation delay through the wireless channel. Therefore, $Y_{s}(f)$ can be written as (refer to Appendix A for derivation)

$$
\begin{aligned}
Y_{s}(f) \approx & X(f) \eta_{T} \sqrt{1-\left|S_{11}(f)\right|^{2}} H_{F}(f) \\
& \times \eta_{L} H_{R}(f) A(f) \eta_{R} \sqrt{1-\left|S_{11}(f)\right|^{2}}
\end{aligned}
$$

where $\eta_{T}$ and $\eta_{R}$ account for the antenna gain and other losses associated with the transmission and reception of signals, respectively. Since the same antenna is used for both transmission and reception, we have $\eta_{T}=\eta_{R}=\eta$. The factor $\eta_{L}$ accounts for the amount of signal reflected back towards the reader due to the reflection at the tag, $H_{F}(f)$ is the forward wireless channel, $H_{R}(f)$ is the backward wireless channel, and $A(f)$ accounts for the effective antenna aperture. Here, the signal being emitted into the wireless medium by the antenna is approximated to be proportional to $\sqrt{\left(1-\left|S_{11}(f)\right|^{2}\right)}$. When (6) is inspected from left to right, it simply expresses how the signal injected into the reader transmit antenna, $X(f)$, propagates through the total system to produce $Y_{s}(f)$, that is, the signal injected to the reader antenna $X(f)$ is emitted by the antenna, propagates through the forward wireless channel $H_{F}(f)$, reaches the tag and gets reflected with some loss, propagates back through the backward wireless channel $H_{R}(f)$, and is received by the same antenna. We assume that the wireless channel between the transmit antenna and the tag is free of multipath propagation and that there exists a direct unobstructed line of sight path between them. Hence, the effect of the wireless channel constitutes simply to an attenuation due to the path loss and a propagation delay of $\tau=R / c$, where $c$ is the speed of light and $R$ is the distance between the reader antenna and tag. Since both forward and backward wireless channels are identical we have

$$
H_{F}(f)=H_{R}(f)=H(f)=\frac{1}{\sqrt{4 \pi} R} e^{-j 2 \pi f \frac{R}{c} t} .
$$

The effective aperture of an antenna is defined as $A_{e}=$ $G\left(\lambda^{2}\right) /(4 \pi)$ when considering a transmission power-based analysis [18]. However, since we are considering the voltage in this analysis, the transfer function $A(f)$ was defined to capture the effect of the effective antenna aperture as follows:

$$
A(f)= \begin{cases}\frac{c}{\sqrt{4 \pi}|f|}\left[\Pi\left(\frac{f-f_{c}}{B}\right)+\Pi\left(\frac{f+f_{c}}{B}\right)\right], & f \neq 0 \\ 0, & f=0\end{cases}
$$

where $B$ is the bandwidth of the antenna, $f_{c}$ is the center frequency of the antenna, and $\Pi(\cdot)$ is the rect function [22], [23]. By taking the inverse Fourier transform of $(6), y_{s}(t)$ can be calculated.

\section{B. Antenna Mode of Tag Backscatter}

The antenna mode of the backscatter results from the signals that are harvested or received by the individual patch antennas of the multipatch chipless RFID tag. Since these patch antennas are terminated with an open circuit (refer to Section III-B), the received signals experience a large mismatch at this termination point. This causes these signals to be reflected once they have reached the termination and get retransmitted back through the patch antennas causing a secondary backscatter (antenna mode) that follows the initial structural mode. Since the chipless tag consists of multiple patch antennas, each individual patch antenna will contribute to the formation of the total antenna mode backscatter. Hence, we can write $y_{a}(t)$ as

$$
y_{a}(t)=\sum_{i=1}^{N} y_{a}^{i}(t)
$$

where $y_{a}^{i}(t)$ is the antenna mode backscatter due to the $i$ th patch antenna of the chipless tag, $i=1, \ldots, N$, having $N$ patch antennas. Similar to (6), an expression for $Y_{a}^{i}(f)$ can be written as follows:

$$
\begin{aligned}
Y_{a}^{i}(f) \approx & X(f) \eta_{T} \sqrt{\left[1-\left|S_{11}(f)\right|^{2}\right]} H_{F}(f) A_{i}(f) \eta_{R}^{i} \\
& \times \sqrt{\left[1-\left|S_{11}^{i}(f)\right|^{2}\right]} \Gamma_{i}(f) \sqrt{\left[1-\left|S_{11}^{i}(f)\right|^{2}\right]} \\
& \times \eta_{T}^{i} H_{R}(f) A(f) \eta_{R} \sqrt{\left[1-\left|S_{11}(f)\right|^{2}\right]}
\end{aligned}
$$

where $\eta_{T}^{i}=\eta_{R}^{i}=\eta^{i}$ accounts for the antenna gain and other losses associated with the $i$ th patch antenna of the tag, and $S_{11}^{i}(f)$ and $\Gamma_{i}(f)$ are, respectively, the return loss profile and the load reflection coefficient of the $i$ th patch antenna. Similar to (8), the transfer function $A_{i}(f)$ accounts for the effect of the effective antenna aperture of the $i$ th patch antenna and is defined as follows:

$$
A_{i}(f)= \begin{cases}\frac{c}{\sqrt{4 \pi^{\prime}} f \mid}\left[\Pi\left(\frac{f-f_{i}}{B_{i}}\right)+\Pi\left(\frac{f+f_{i}}{B_{i}}\right)\right], & f \neq 0 \\ 0, & f=0\end{cases}
$$

where $B_{i}$ and $f_{i}$ are the bandwidth and center frequency of the $i$ th patch antenna, respectively. A general expression for $\Gamma_{i}(f)$ can be written as [6]

$$
\Gamma_{i}(f)=\Gamma_{L} \exp \left(-j 2 \pi f 2 \tau_{i}\right)
$$

In this work, the individual patch antennas are terminated abruptly using an open circuit $\Gamma_{L}=1$. The propagation delay from the point where the radiation is picked up by the antenna to the point of termination is defined by $\tau_{i}$.

\section{Total Received Signal}

Using (1), (2), and (9), the Fourier transform of the total received signal $y(t)$ can be written as

$$
Y(f)=S_{11}(f) X(f)+Y_{s}(f)+\sum_{i=1}^{N} Y_{a}^{i}(f)
$$

An approximation for $Y(f)$ can be obtained by substituting for $Y_{s}(f)$ and $Y_{a}^{i}(f)$ using (6) and (10). The received time-domain signal $y(t)$ can be approximated by taking the inverse Fourier transform of (13).

\section{Results Based on Semi-Analytical Model}

Using (13) and substituting for the return loss profiles of the reader's transmit/receive antenna and the tag's patch antennas 


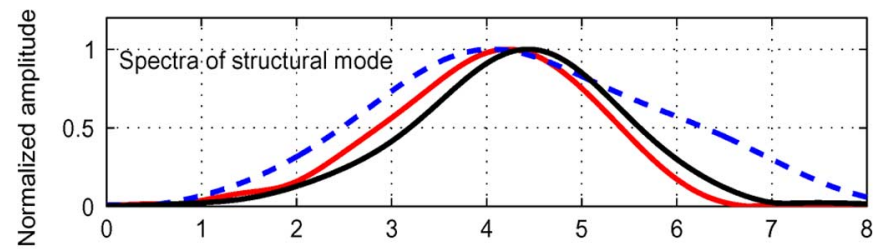

(a)

Frequency $(\mathrm{GHz})$

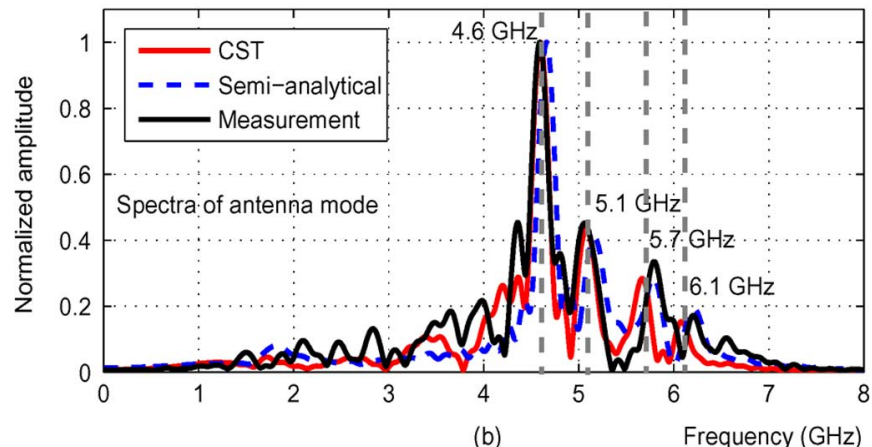

Fig. 6. (a) Normalized amplitude spectrum of $y_{a}(t)$ (b) Normalized amplitude spectrum of $y_{s}(t)$.

using the simulated $S_{11}(f)$ obtained through CST, an approximation for the total received signal in the time domain was obtained. The structural mode and the antenna mode of the backscatter were windowed using a raised cosine window as in Section III-C. The spectral content of the antenna mode and the structural mode is shown in Fig. 6(a) and (b), respectively. There is a close agreement between the CST results and the results obtained through the semi-analytical approximation where four distinct peaks in the spectral signature are clearly visible at the resonance frequencies of the four tag patch antennas.

\section{EXPERIMENTAL VALIDATION}

Here, we will discuss the experimental validation of the simulation results observed previously. For this purpose, experiments were performed in an anechoic chamber enviroment. The experiments were conducted using a vector network analyzer (Agilent PNA E8361A) where the measurements were taken in the frequency domain. These measured data were then converted to the time domain using signal-processing techniques.

\section{A. Transmit/Receiving Reader Antenna and Chipless RFID Tag}

The interrogation signals were transmitted and received by the reader using a single monopole antenna. Fig. 7(a) shows the antenna used for the experiment. It is a CPW-fed circular disc loaded monopole antenna with a $0.3-\mathrm{mm}$ gap between the CPW feed line and the ground. The reader antenna was fabricated on a Taconic TLX-8 substrate material having thickness of $0.5 \mathrm{~mm}$ with copper cladding thickness of $17 \mu \mathrm{m}$. The measured return loss and the E-field radiation patterns for the antenna are shown in Fig. 8(a) and (b), respectively. The antenna performs well from 1.5 to $5 \mathrm{GHz}$. The return loss profile degrades after $5 \mathrm{GHz}$. The radiation pattern is omnidirectional for lower frequencies and becomes directive at higher frequencies. The chipless RFID tag used in the experiment is shown in Fig. 7(b). Its operation is explained in detail in Section III-B.

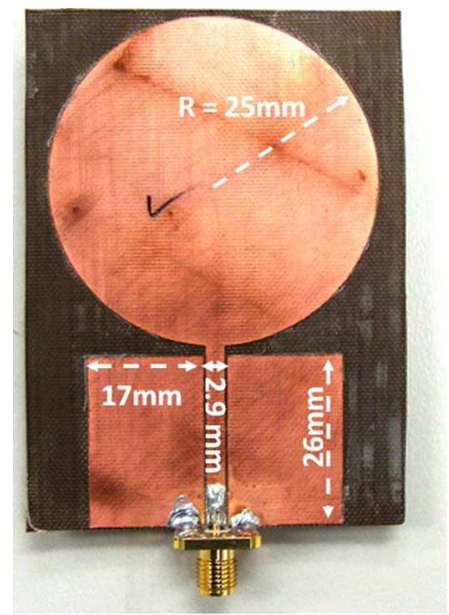

(a)

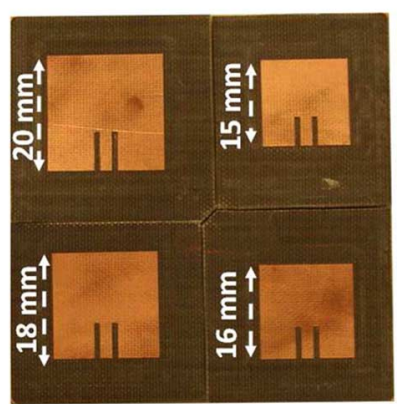

(b)
Fig. 7. (a) Transmit/receive antenna: coplanar circular disc loaded monopole antenna. (b) Chipless RFID tag consisting of four inset-fed square patches. Both antenna and tag are fabricated on Taconic TLX- 8 having a substrate thickness of $0.5 \mathrm{~mm}$, copper thickness of $17 \mu \mathrm{m}$, and dielectric constant of 2.55. The gap between the feed line and the ground of the antenna is $0.3 \mathrm{~mm}$.

\section{B. Measurement Setup and Results}

Measurements were taken in an anechoic chamber where a single-port measurement was carried out using a VNA with a transmission power of $1 \mathrm{~mW}$. Fig. 9 shows the placement of the tag and the reader antenna inside the chamber. The experiment involves two steps. First, the loaded return loss profile of the antenna, $S_{11}^{\text {Loaded }}$, was measured where the presence of the tag affects the return loss profile of the reader antenna. Next, the unloaded return loss of the antenna $S_{11}$ was measured with an empty chamber without the tag. By applying (4) in Section II to these experimental frequency-domain measurements, the time-domain backscatter from the tag $y_{s}(t)+y_{a}(t)$ was obtained. Using a raised cosine window, the signals $y_{s}(t)$ and $y_{a}(t)$ are windowed as described in Section III-C. The amplitude spectra of these windowed $y_{s}(t)$ and $y_{a}(t)$ are shown in Fig. 6, where the chipless tag was placed $30 \mathrm{~cm}$ away from the reader antenna in the measurement setup shown in Fig. 9. It is clear that the measurement results are in accordance with the simulation results and the semi-analytical results. It should be noted that the results obtained did not rely on the use of a calibration tag.

\section{Tag Performance in Different Orientations}

The performance of the proposed technique was tested experimentally where the tag was placed in different orientations and locations with respect to the reader antenna. Fig. 10 shows the frequency spectra of chipless tags having different combinations of resonant patch antennas $\left(f_{1}=4.6 \mathrm{GHz}, f_{2}=5.1 \mathrm{GHz}\right.$, $f_{3}=5.8 \mathrm{GHz}$, and $f_{4}=6.2 \mathrm{GHz}$ ). The result confirms that the presence of a resonant patch antenna in the chipless tag causes a corresponding peak in the spectral signature of the chipless tag.

The performance of the chipless RFID system at different distances is shown in Fig. 11. A tag having resonant patch antennas at frequencies $f_{1}$ and $f_{3}$ was positioned at $R=15$, 30 , and $50 \mathrm{~cm}$ away from the reader antenna. The amplitude 


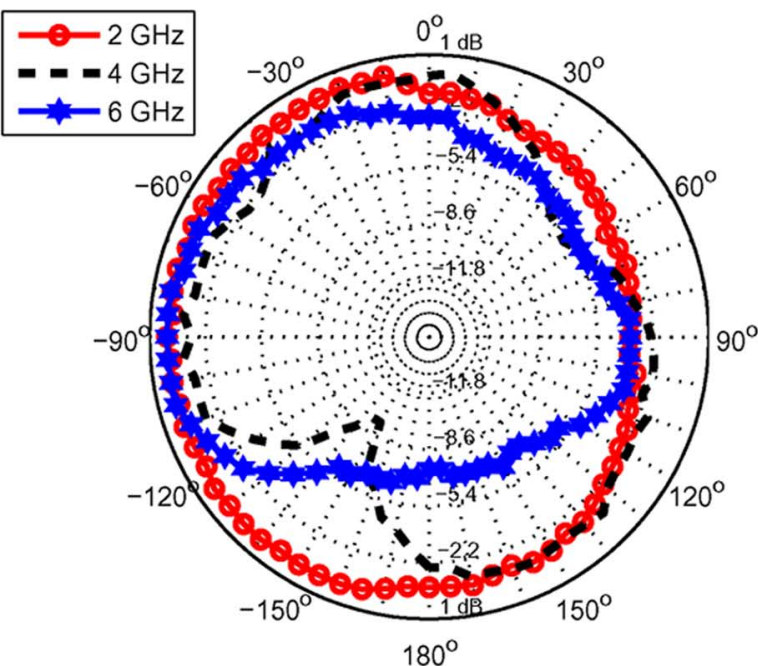

(a)

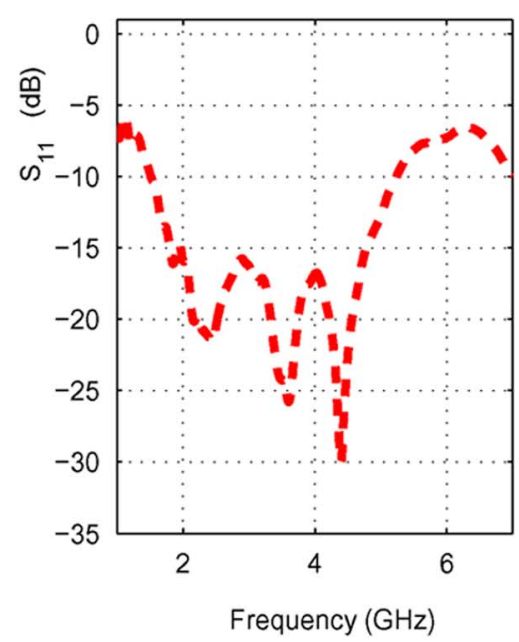

(b)

Fig. 8. (a) Normalized measured E-field radiation pattern of antenna. (b) Measured return loss of antenna.

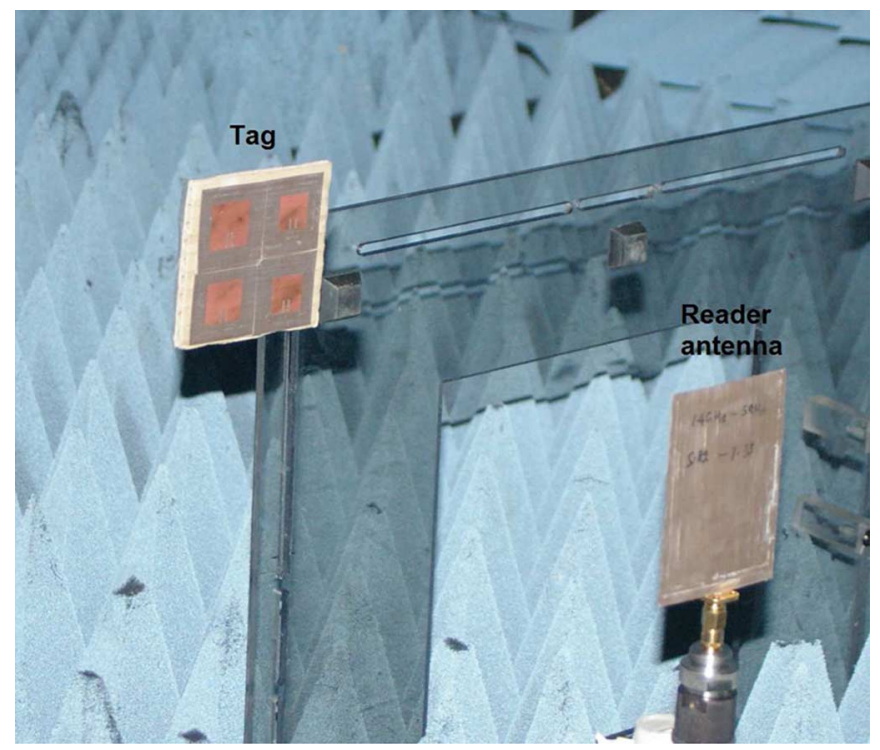

Fig. 9. Experimental setup for testing chipless RFID system in anechoic chamber environment.

spectrum of the antenna mode backscatter for the different distances is shown in the figure where the amplitudes are normalized with respect to the maximum amplitude registered with the $R=15-\mathrm{cm}$ measurement. It is clearly observed that the amplitude of the spectrum reduces as the distance increases whereas the shape is preserved. In all three measurements, two distinct spectral peaks are clearly visible at the resonance frequencies $f_{1}$ and $f_{3}$. It is also observed that, as the distance increases, the signal-to-noise ratio (SNR) degrades, which causes ambiguity in the detection of resonant peaks in the spectral signature at higher frequencies particularly with the $R=50 \mathrm{~cm}$ reading.

The tag was rotated about its axis of symmetry as shown in Fig. 12(a). The rotation was done about the $z$-axis such that the patch antennas corresponding to the higher frequencies would move away from the reader antenna while the patch antennas corresponding to lower frequencies would move toward the

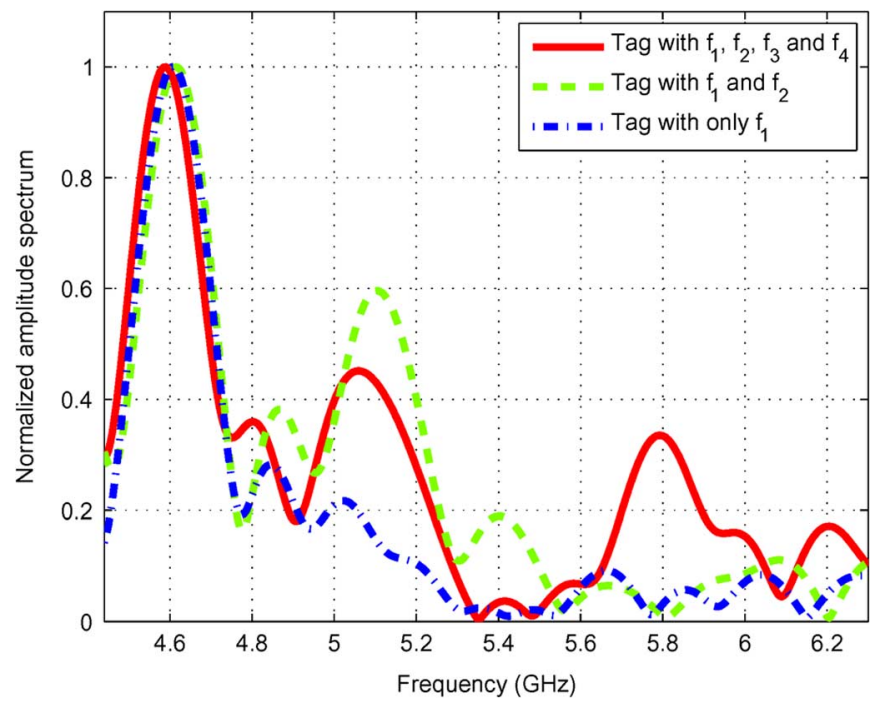

Fig. 10. Frequency spectra of different chipless tags placed $30 \mathrm{~cm}$ in front of the reader antenna.

reader antenna. Fig. 13 shows the estimated tag frequency spectra when the tag is rotated. The tag contained resonant patch antennas at $f_{1}, f_{2}$, and $f_{3}$, where it was placed $30 \mathrm{~cm}$ in front of the reader antenna. It is clear from the results that for rotations less than $45^{\circ}$ the spectral signature of the chipless tag can be estimated using the proposed technique without any additional signal processing. All three resonant frequencies of the tag can be clearly distinguished. However, when the tag is rotated beyond $45^{\circ}$ the performance degrades and some of the higher resonant frequencies do not appear in the estimated frequency spectra.

Since the tag consists of microstrip patch antennas, it has a fairly directive radiation pattern (ranging from 8 to $8.7 \mathrm{dBi}$ ). As illustrated in Fig. 12(b), when the tag rotates about the $z$-axis, the main lobe of its radiation pattern faces away from the reader antenna. Therefore, the amplitude of the antenna mode frequency spectra gradually reduces as the tag is rotated. We 


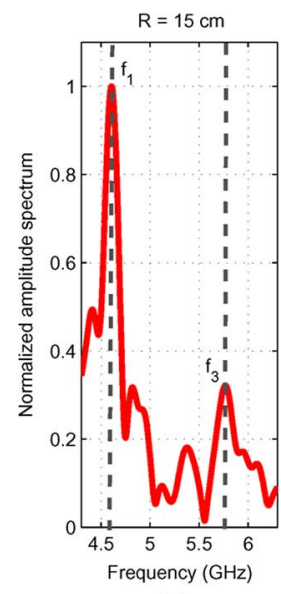

(a)

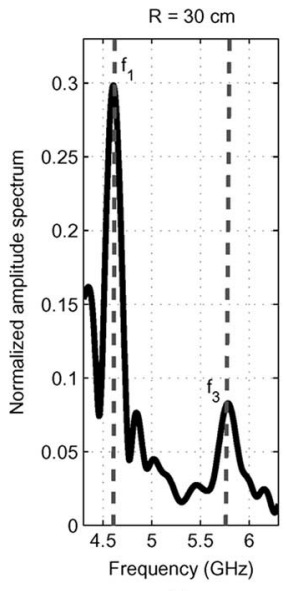

(b)

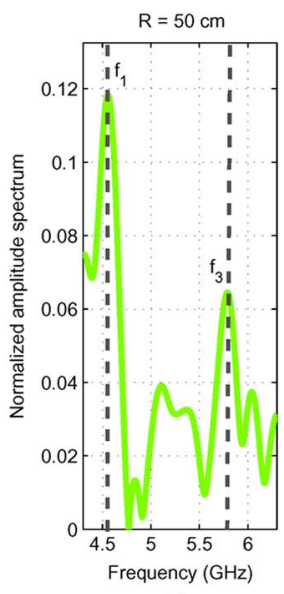

(c)
Fig. 11. Frequency spectra of a tag with resonant patch antennas at frequencies $f_{1}$ and $f_{3}$ placed at different distances from the reader antenna. All of the amplitudes are normalized with respect to the maximum amplitude of the antenna mode observed with the $R=15-\mathrm{cm}$ measurement.
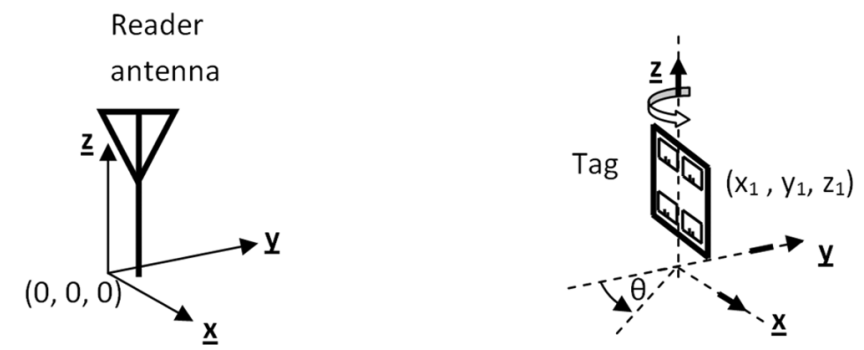

(a)

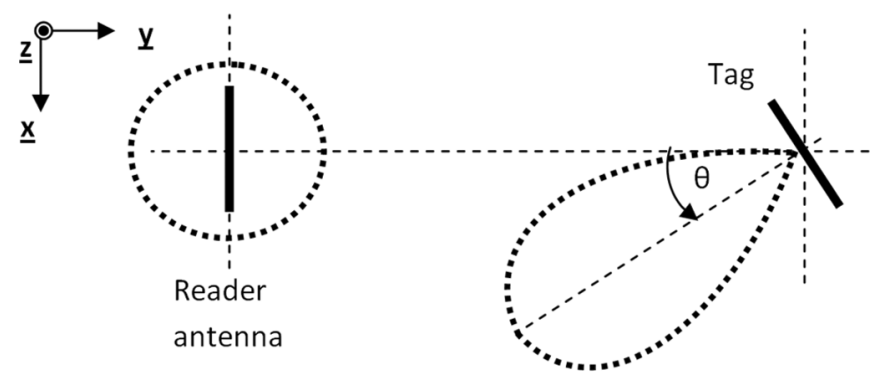

(b)

Fig. 12. (a) Perspective view of the position of the tag with respect to the reader antenna. (b) View of the $x-y$ plane, showing the radiation patterns of the tag and the reader antenna.

hypothesize that the amplitude spectrum of the antenna mode does not experience a large change when the rotation angle $\theta$ is such that $\left(\theta=20^{\circ}, 30^{\circ}\right)$, the portion of the tag radiation pattern directed towards the reader antenna is still within the half-power beam-width (HPBW) of the tag radiation pattern. However, as soon as $\theta$ exceeds this threshold (HPBW/2), the observed spectrum will be severely affected.

Due to the tag rotation, a frequency shift is also observed in the spectral peaks, particularly in the peak corresponding to $f_{1}$. We speculate that this is a result of the complex interaction of both the rotation of the main radiation lobe of the tag, as mentioned earlier, and the directive radiation pattern of the reader antenna at higher frequencies as shown in Fig. 8(a). When a

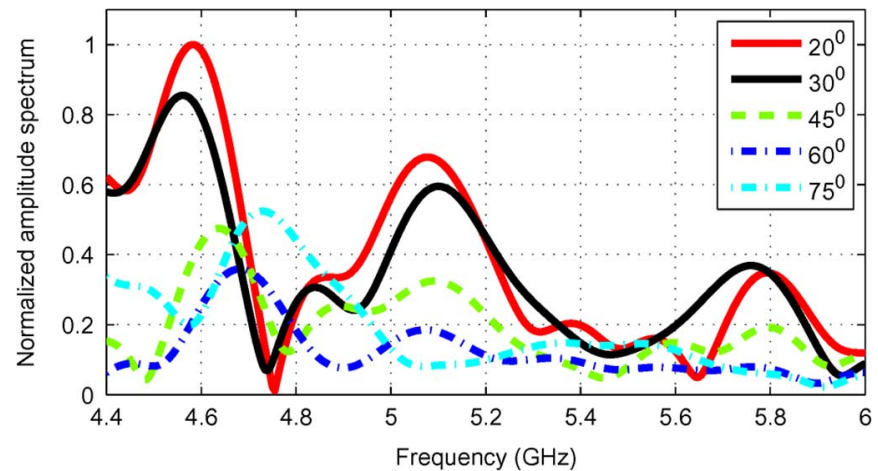

Fig. 13. Frequency spectra of a tag having resonances $f_{1}, f_{2}$, and $f_{3}$ placed $30 \mathrm{~cm}$ away from the reader antenna. The tag is rotated about itself (about the $z$-axis) at different angles. The amplitude is normalized with respect to the maximum amplitude of the amplitude spectrum obtained for the unrotated or $0^{\circ}$ position.

sufficiently large guard band (200 MHz) is utilized between the resonant frequencies, the effect of this shift on the detection performance can be minimized to some extent.

\section{DATA REPRESENTATION AND DETECTION}

Here, we discuss the representation of digital information using the spectral content of the antenna mode backscatter and on the detection of information bits contained in the backscatter of a chipless tag.

\section{A. Data Representation}

From the measurement results presented in the previous section, it is clear that the proposed method of analyzing the spectral content of the windowed antenna mode backscatter reveals the resonant features of the chipless RFID tag. With the prototype tag consisting of four distinct patch antennas, it is possible to encode four data bits where the presence of a patch represents a " 1 " bit and its absence signifies a " 0 " bit, that is, we consider a resonant peak observed in the frequency spectrum of the antenna mode backscatter to denote a logic "1." If we encode the most significant bit (MSB) as the lowest frequency $f_{1}$ and the least significant bit (LSB) as the highest frequency $f_{4}$, then all of the spectral signatures shown in Fig. 11 encode the data "1010."

\section{B. Detection of Information Bits}

From the results shown in Section V, we can observe that, when the distance to the tag increases or when the tag is rotated, the amplitudes of the resonant peaks reduces and the SNR reduces. Therefore, the detection of these peaks is not that obvious, and false peaks due to noise and clutter introduce ambiguity in the detection process. The use of a fixed threshold based detection scheme as in [8] is not justifiable in this context. This is because the amplitude spectrum of the interrogation pulse $x(t)$ is not uniform; the center frequencies have higher amplitudes while the lower and higher frequencies have very low amplitudes due to the Gaussian-shaped amplitude spectrum. Therefore, additional signal processing is required for the accurate detection of the information contained in the spectral signature of the chipless tag. For this purpose, we propose a 


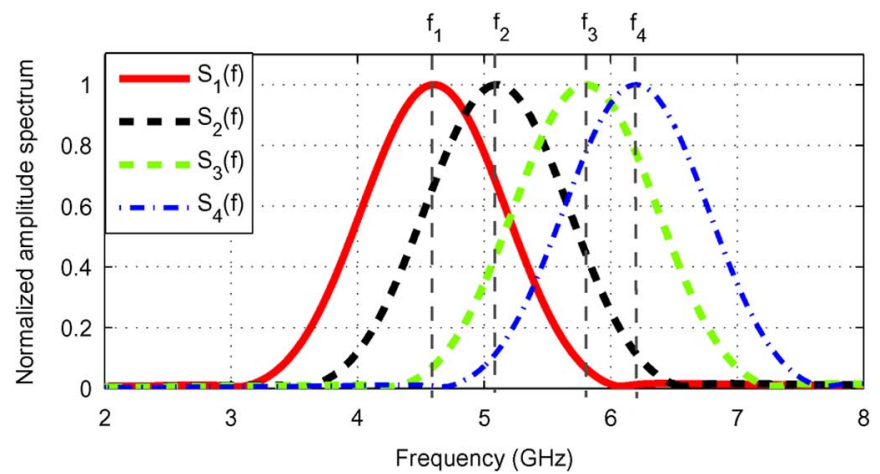

Fig. 14. Amplitude spectra of the set of pulses $s_{i}(t), i=1, \ldots, 4$.

novel technique, selective spectral interrogation (SSI), for detection which is based on using a set of interrogation pulses, $s_{i}(t), i=1, \ldots, 4$ defined as follows:

$s_{i}(t)=A_{i} \cos \left(2 \pi f_{i} t\right) \exp \left(-\frac{\left(t-\mu_{2}\right)^{2}}{2 \sigma_{2}^{2}}\right), \quad i=1, \ldots, 4$

where $A_{i}=1, i=1, \ldots, 4, \mu_{2}=0.6 \mathrm{~ns}$, and $\sigma_{2}=0.3 \mathrm{~ns}$. Here, the carrier frequency of the modulated Gaussian pulse is chosen to be the resonance frequencies of the patch antennas $f_{i}, i=1, \ldots, 4$. The parameter $\sigma_{2}$ of the Gaussian pulse is larger than that of (5), which makes this pulse less broadband with more energy concentrated around $f_{i}$. The premise behind the operation of this method is as follows. When the tag is interrogated with the pulse $s_{j}(t), i=j$, if the tag contains a resonant patch antenna at $f_{j}$, then the amplitude spectrum of the antenna mode backscatter should show a spectral peak at $f_{j}$. This peak will be considerably larger than all of the other peaks corresponding to the other resonance frequencies of the tag or false peaks due to noise. This is because the pulse $s_{i}(t)$ contains more energy near $f_{j}$ than all of the other resonance frequencies, hence causing the tag resonance at $f_{j}$ to be more prominent if it exists. If such a maxima in the amplitude spectrum is not observed, then it implies that the tag does not contain the patch antenna resonating at $f_{j}$. Fig. 14 shows amplitude spectra $\left|S_{i}(f)\right|$ of the four different interrogation pulses $s_{i}(t)$. The 20-dB bandwidth of these pulses are approximately $2.2 \mathrm{GHz}$. By substituting $X(f)$ with $S_{i}(f)$ in (4), the backscatter response from the tag when the tag is interrogated using the pulse $s_{i}(t)$ can be calculated from the measured $S_{11}(f)$ data.

Fig. 15 shows the frequency spectrum of the windowed antenna mode backscatter when a tag having patch antennas resonating at $f_{1}$ and $f_{3}$ was interrogated using the pulses $s_{i}(t)$. The tag was placed $50 \mathrm{~cm}$ away from the reader antenna [Fig. 11(c) shows the frequency spectrum of this tag when interrogated using the UWB pulse defined in (5)]. From the figure, it is clear that, when $s_{1}(t)$ and $s_{3}(t)$ are used to interrogate the tag, the maximum amplitude occurs at the corresponding resonance frequencies of $f_{1}$ and $f_{3}$, whereas, when the pulses $s_{2}(t)$ and $s_{4}(t)$ are used, the maximum amplitude does not occur at the respective resonances of $f_{2}$ and $f_{4}$. This confirms that the tag does not contain the patch antennas that resonate at $f_{2}$ and $f_{4}$. Therefore, the information contained in the tag is correctly detected as "1010."

The frequency spectra of the antenna mode backscatter obtained for a tag having patch antennas resonating at $f_{1}$ and $f_{4}$ is

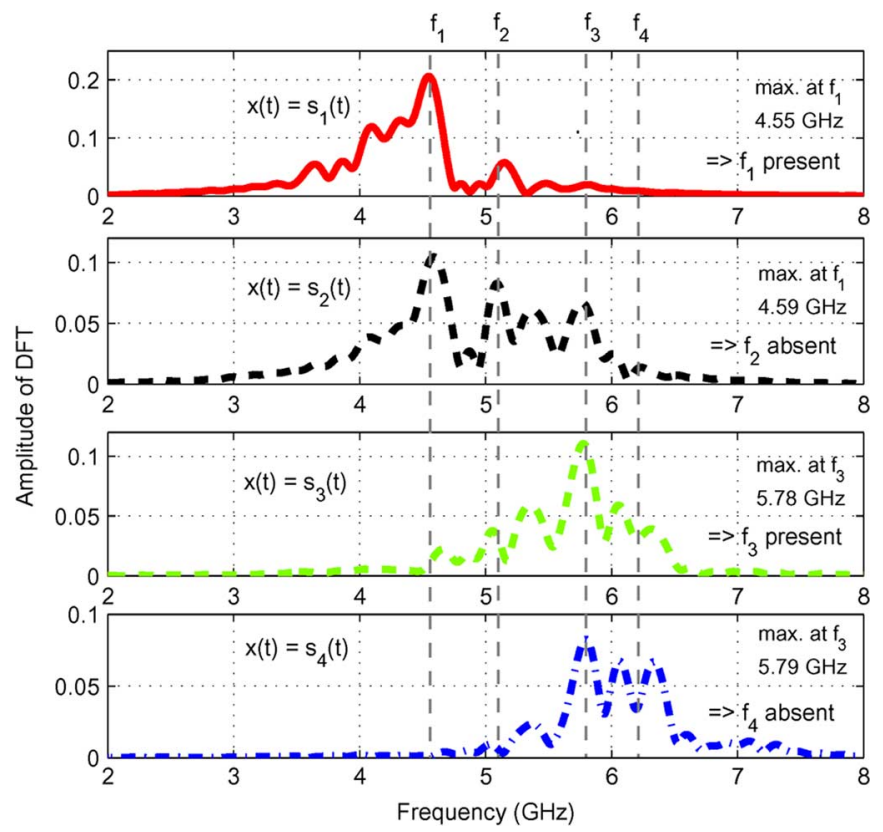

Fig. 15. Amplitude spectrum of the windowed antenna mode backscatter for different pulses $s_{i}(t)$. The tag measured contained patch antennas resonating at $f_{1}$ and $f_{3}$ (tag carried data "1010") and was placed $50 \mathrm{~cm}$ away from the reader antenna.

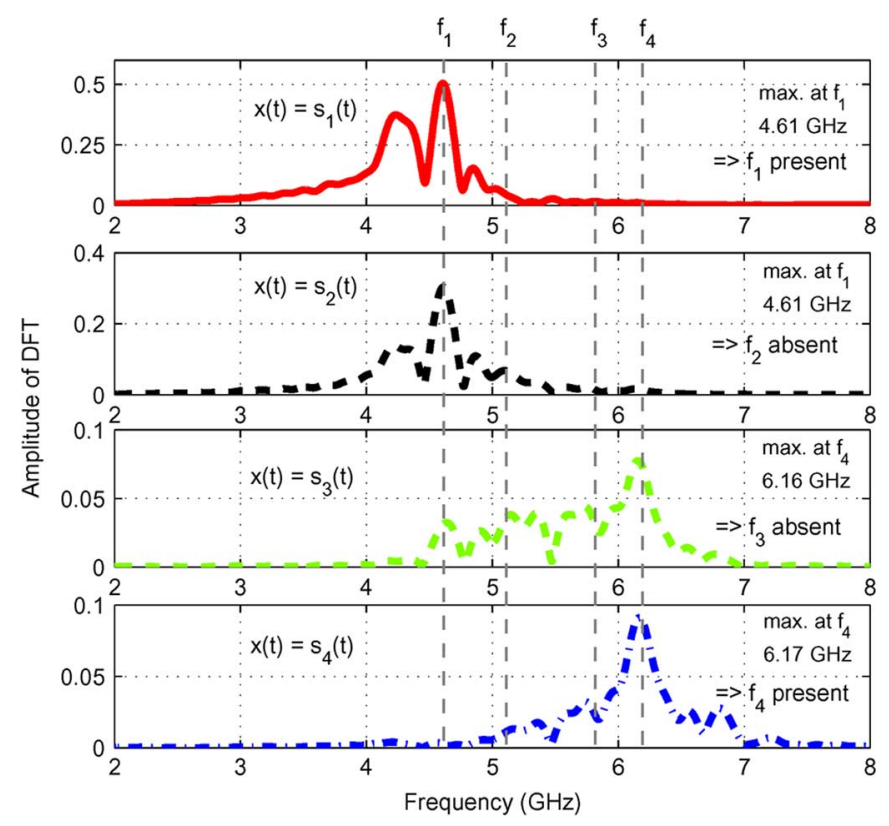

Fig. 16. Amplitude spectrum of the windowed antenna mode backscatter for different pulses $s_{i}(t)$. The tag measured contained patch antennas resonating at $f_{1}$ and $f_{4}$ (tag carried data " 1001 ") and was placed $30 \mathrm{~cm}$ away from the reader antenna.

shown in Fig. 16. Here, when the pulses $s_{2}(t)$ and $s_{3}(t)$ are used, the maximum amplitude does not occur at $f_{2}$ and $f_{3}$, which confirms that neither of these resonances are present in the tag. Hence, the information contained in the tag can be correctly detected as "1001."

It is clear that, by using the SSI method, the data contained in the amplitude spectrum of the antenna mode backscatter can be extracted using a peak detection algorithm. The performance of this algorithm can be further enhanced by supplementing the 
peak detection with an energy computation metric that provides the energy at the vicinity of each resonance frequency.

\section{CONCLUSION}

In this paper, a new method is proposed to accurately estimate the resonant features of a multipatch frequency-spectra-based chipless RFID tag. A UWB-IR-based reader architecture is used to interrogate the tag, where the backscatter is analyzed in the time domain. Through electromagnetic simulations, it is shown that the information-carrying component of the received signal is contained in the antenna mode of the backscatter, whereas the structural mode of the backscatter contains no information about the resonant properties of the chipless tag. A semi-analytical approximation is also derived to explain the nature of the backscattered signal and to provide insight on how each and every individual component of the system contributes to the total received signal at the reader. The results obtained from simulations and the semi-analytical approximation are also validated by measurement results obtained through experiments conducted in an anechoic chamber environment. The proposed method is capable of successfully estimating the resonant properties of a chipless tag without using calibration tags and additional signal processing up to a distance of $30 \mathrm{~cm}$. Also, the effects of tag orientation and location with respect to the reader on the estimation of the tag frequency spectra is investigated. It is shown that, for tag rotations less than $45^{\circ}$, the estimated frequency spectra is usable for detecting the information contained in it. A novel detection method is introduced to perform detection of the spectral peaks amidst false peaks due to noise and clutter. The method uses the tag responses obtained for a set of interrogation pulses and a peak searching algorithm. It is shown that, by applying the new method on the measured data, it is possible to enhance the read range of a tag up to $50 \mathrm{~cm}$.

\section{APPENDIX}

Here, we present the derivation of the semi-analytical approximation for $Y_{s}(t)$. First, consider the radar range equation for a bi-static radar given in [18, (2)-(125)]:

$$
\begin{aligned}
\frac{P_{r}}{P_{t}}= & e_{t}\left(1-\left|S_{11}(f)\right|^{2}\right) \frac{D_{t}\left(\theta_{t}, \phi_{t}\right)}{4 \pi R_{1}^{2}} \\
& \times \sigma \frac{\lambda^{2}}{4 \pi} \frac{D_{r}\left(\theta_{r}, \phi_{r}\right)}{4 \pi R_{2}^{2}} e_{r}\left(1-\left|S_{22}(f)\right|^{2}\right)
\end{aligned}
$$

where $P_{r}$ and $P_{t}$ are the received and transmitted power, respectively, $S_{11}(f)$ is the return loss of the transmitting antenna, $S_{22}(f)$ is the return loss of the receiving antenna, $e_{t}$ and $e_{r}$ are the losses associated with transmission and reception, respectively, $D_{t}\left(\theta_{t}, \phi_{t}\right)$ is the directivity of the transmit antenna, $D_{r}\left(\theta_{r}, \phi_{r}\right)$ is the directivity of the receiving antenna, $R_{1}$ is the distance from the transmit antenna to the target, $R_{2}$ is the distance from the target to the receiving antenna, $\lambda$ is the wavelength, and $\sigma$ is the radar cross section of the target being considered. We assume that there is no polarization mismatches between the antennas. In the case of the chipless RFID system shown in Fig. 1 the same antenna serves as both the transmitting and receving antenna. Therefore, we have $S_{11}(f)=S_{22}(f)$, and $R_{1}=R_{2}=R$. For the purpose of deriving an expression for $Y_{s}(f)$ we consider the tag to be a metallic object having no resonant properties. Therefore, it does not give rise to an antenna mode, $Y_{a}(f)$, where the lossy reflection received by the antenna consists of only the structural mode $Y_{s}(f)$ which is simply an attenuated and delayed version of the signal being transmitted. By taking the square root of (15), we can obtain the magnitude of the voltage induced at the receiver, $\left|Y_{s}(f)\right|$, when $X(f)$ is transmitted as follows:

$$
\begin{aligned}
\frac{\left|Y_{s}(f)\right| / \sqrt{Z_{0}}}{|X(f)| / \sqrt{Z_{0}}}= & \sqrt{\left(1-\left|S_{11}(f)\right|^{2}\right)} \sqrt{e_{t} \frac{D_{t}\left(\theta_{t}, \phi_{t}\right)}{4 \pi R^{2}}} \\
& \times \sqrt{\sigma} \sqrt{\frac{\lambda^{2}}{4 \pi}} \sqrt{e_{r} \frac{D_{r}\left(\theta_{r}, \phi_{r}\right)}{4 \pi R^{2}}} \\
& \times \sqrt{\left(1-\left|S_{11}(f)\right|^{2}\right)}
\end{aligned}
$$

where $Z_{0}$ is the characteristic impedance of the antenna. Equation (16) only gives the magnitude of the induced voltage at the antenna terminals. By including the propagation delay $\tau=$ $(R) /(c)$ introduced to the signal when it is propagating through the forward and backward wireless channels, a part of the phase information contained in $Y_{s}(f)$ can be restored as

$$
\begin{aligned}
Y_{s}(f) \approx & X(f) \sqrt{\left(1-\left|S_{11}(f)\right|^{2}\right)} \\
& \times \sqrt{e_{t} D_{t}\left(\theta_{t}, \phi_{t}\right)} \frac{1}{\sqrt{4 \pi} R} e^{-j 2 \pi f \frac{R}{c} t} \\
& \times \sqrt{\sigma} \frac{c}{\sqrt{4 \pi}|f|} \sqrt{e_{r} D_{r}\left(\theta_{r}, \phi_{r}\right)} \frac{1}{\sqrt{4 \pi} R} e^{-j 2 \pi f \frac{R}{c} t} \\
& \times \sqrt{\left(1-\left|S_{11}(f)\right|^{2}\right)} .
\end{aligned}
$$

However, (17) serves only as an approximation because it neglects the phase transformations introduced due to $S_{11}(f)$ when the signal is being transmitted and received via the antenna. Equation (17) can be rewritten as

$$
\begin{aligned}
Y_{s}(f) \approx & X(f) \eta_{T} \sqrt{1-\left|S_{11}(f)\right|^{2}} H_{F}(f) \\
& \times \eta_{L} H_{R}(f) A(f) \eta_{R} \sqrt{1-\left|S_{11}(f)\right|^{2}}
\end{aligned}
$$

where $\eta_{T}=\sqrt{e_{t} D_{t}\left(\theta_{t}, \phi_{t}\right)}, \eta_{R}=\sqrt{e_{r} D_{r}\left(\theta_{r}, \phi_{r}\right)}, H_{F}(f)=$ $H_{R}(f)=(1) /(\sqrt{4 \pi} R) e^{-j 2 \pi f(R) /(c) t}$, and $\eta_{L}=\sqrt{\sigma}$. The term $(c) /(\sqrt{4 \pi}|f|)$ due to the effective aperture of the receive antenna is captured by the function $A(f)$, which is defined in (8). This function simply captures the effect of the effective antenna aperture for the entire bandwidth of operation of the antenna.

\section{REFERENCES}

[1] S. Preradovic and N. C. Karmakar, "Chipless RFID: Bar code of the future," IEEE Microw. Mag., vol. 11, no. 7, pp. 87-97, Dec. 2010.

[2] L. Zhang, S. Rodriguez, H. Tenhunen, and L.-R. Zheng, "An innovative fully printable RFID technology based on high speed time-domain reflections," in Proc. Conf. HDP, Jun. 2006, pp. 166-170.

[3] A. Chamarti and K. Varahramyan, "Transmission delay line based ID generation circuit for RFID applications," IEEE Microw. Wireless Compon. Lett., vol. 16, no. 11, pp. 588-590, Nov. 2006.

[4] L. Zheng, S. Rodriguez, L. Zhang, B. Shao, and L.-R. Zheng, "Design and implementation of a fully reconfigurable chipless RFID tag using inkjet printing technology," in Proc. IEEE Int. Symp. Circuits Syst., May 2008, pp. 1524-1527.

[5] B. Shao, Q. Chen, Y. Amin, S. M. David, R. Liu, and L.-R. Zheng, "An ultra-low-cost RFID tag with $1.67 \mathrm{gbps}$ data rate by ink-jet printing on paper substrate," in Proc. IEEE Asian Solid State Circuits Conf., Nov. 2010, pp. 1-4.

[6] A. Lazaro, A. Ramos, D. Girbau, and R. Villarino, "Chipless UWB RFID tag detection using continuous wavelet transform," IEEE Antennas Wireless Propagat. Lett., vol. 10, pp. 520-523, 2011. 
[7] A. Ramos, D. Girbau, A. Lazaro, and S. Rima, "IR-UWB radar system and tag design for time-coded chipless RFID," in Proc. 6th Eur. Conf. Antennas and Propagation, Mar. 2012, pp. 2491-2494.

[8] S. Preradovic and N. C. Karmakar, "Design of short range chipless RFID reader prototype," in Proc. 5th Int. Conf. Intell. Sensors, Sensor Networks and Inf. Process., Melbourne, Australia, Dec. 2009, pp. 307-312.

[9] I. Balbin and N. C. Karmakar, "Phase-encoded chipless RFID transponder for large-scale low-cost applications," IEEE Microw. Wireless Compon. Lett., vol. 19, no. 8, pp. 509-511, July 2009.

[10] P. Kalansuriya, N. Karmakar, and E. Viterbo, "Signal space representation of chipless RFID tag frequency signatures," in Proc. IEEE Global Telecommun. Conf., Houston, TX, Dec. 2011, pp. 1-5.

[11] R. V. Koswatta and N. C. Karmakar, "A novel reader architecture based on UWB chirp signal interrogation for multiresonator-based chipless RFID tag reading," IEEE Trans. Microw. Theory Tech., vol. 60, no. 9, pp. 2925-2933, Sep. 2012.

[12] S. Preradovic and N. Karmakar, "Design of fully printable planar chipless RFID transponder with 35-bit data capacity," in Proc. Microw. Conf., Sep. 29-Oct. 12009 , pp. 013-016.

[13] M. Islam and N. Karmakar, "A novel compact printable dual-polarized chipless RFID system," IEEE Trans. Microw. Theory Tech., vol. 60, no. 7, pp. 2142-2151, Jul. 2012.

[14] S. Hu, C. L. Law, and W. Dou, "A balloon-shaped monopole antenna for passive UWB-RFID tag applications," IEEE Antennas Wireless Propag. Lett., vol. 7, pp. 366-368, Jul. 2008.

[15] A. Blischak and M. Manteghi, "Embedded singularity chipless RFID tags," IEEE Trans. Antennas Propag., vol. 59, no. 11, pp. 3961-3968, Nov. 2011.

[16] P. Kalansuriya and N. Karmakar, "Time domain analysis of a backscattering frequency signature based chipless RFID tag," in Asia-Pacific Microw. Conf. Proc., Melbourne, Australia, Dec. 2011, pp. 183-186.

[17] P. Kalansuriya and N. Karmakar, "UWB-IR based detection for frequency-spectra based chipless RFID," in IEEE MTT-S Int. Microw. Symp. Dig., Montreal, QC, Canada, Jun. 2012, pp. 1-3.

[18] C. A. Balanis, Antenna Theory Analysis and Design, 3rd ed. Hoboken, NJ: Wiley, 2005.

[19] N. Karmakar, Handbook of Smart Antennas for RFID Systems. Hoboken, NJ: Wiley, 2010 [Online]. Available: http://books. google.com/books?id=WgcSH4G86YAC

[20] "Complete technology for 3D EM simulation," CST Computer Simulation Technology AG, Darmstadt, Germany. [Online]. Available: http:// www.cst.com

[21] A. Oppenheim, A. Willsky, and S. Nawab, Signals and Systems. Englewood Cliffs, NJ: Prentice-Hall, 1997.

[22] R. Bracewell, The Fourier Transform and Its Applications. New York: McGraw-Hill, 2000.

[23] E. W. Weisstein, "Rectangle function," MathWorld-A Wolfram Web Resource. [Online]. Available: http://mathworld.wolfram.com/RectangleFunction.html

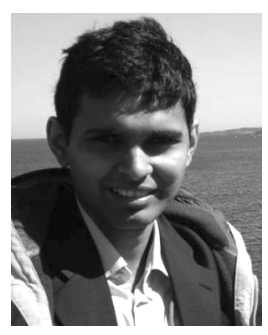

Prasanna Kalansuriya (S'08) received the B.Sc. degree (with first-class honors) from the University of Moratuwa, Moratuwa, Sri Lanka, in 2005, and the M.Sc. degree from the University of Alberta, Edmonton, AB, Canada, in 2009. He is currently working toward the Ph.D. degree in electrical and computer systems engineering at Monash University, Clayton, Australia.

From 2005 to 2007, he was an Electronic Design Engineer with Electroteks Global Networks, Pte Ltd, Sri Lanka. He has served as a Lecturer with the University of Moratuwa, Moratuwa, Sri Lanka, in 2007, and as a Research Assistant with the ICORE Wireless Communication Laboratory, University of Alberta, Edmonton, AB, Canada, from 2008 to 2010. In 2012, he was a Visiting Researcher with the Auto-ID Laboratory, Massachusetts Institute of Technology, Cambridge. His research interests include wireless communication, signal processing for chipless RFID, and applications of chipless RFID and passive RFID in pervasive sensing.

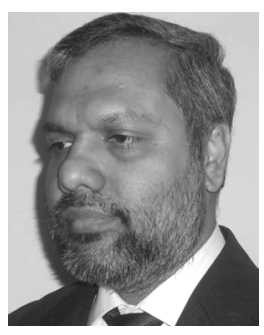

Nemai Chandra Karmakar (S'91-M'91-SM'99) received the M.Sc. degree in electrical engineering from the University of Saskatchewan, Saskatoon, SK, Canada, in 1991, and the Ph.D. degree from the University of Queensland, Brisbane, Australia, in 1999.

He is an Associate Professor with the Department of Electrical and Computer Systems Engineering, Monash University, Clayton, Australia. He possesses approximately 20 years of teaching, design, and development experience in antennas, microwave active and passive circuits, and RFIDs in Canada, Australia, and Singapore. $\mathrm{He}$ has authored or coauthored over 220 referred journal and conference papers, 24 book chapters, and six books. He has two patents (pending) in chipless RFID technology.

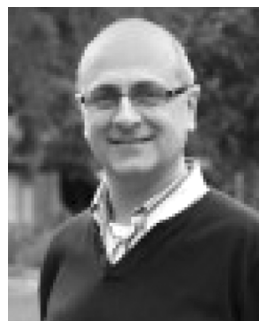

Emanuele Viterbo (M'95-SM'04-F'11) received the Laurea and Ph.D. degrees from the Politecnico di Torino, Torino, Italy, in 1989 and 1995, respectively, both in electrical engineering.

From 1990 to 1992, he was with the European Patent Office, The Hague, The Netherlands, as a Patent Examiner working in the field of dynamic recording and error-control coding. Between 1995 and 1997, he held a postdoctoral position with the Dipartimento di Elettronica, Politecnico di Torino, Torino, Italy. During 1997-1998, he was a Post-Doctoral Research Fellow with the Information Sciences Research Center, AT\&T Research, Florham Park, NJ. He became first an Assistant Professor (1998) and then an Associate Professor (2005) with the Dipartimento di Elettronica at Politecnico di Torino. In 2006, he became a Full Professor with DEIS, University of Calabria, Italy. Since 2010, he has been a Full Professor with the Department of Electrical and Computer Systems Engineering and Associate Dean for Research Training in the Faculty of Engineering, Monash University, Clayton, Australia. In 1993, he was a Visiting Researcher with the Communications Department, DLR, Oberpfaffenhofen, Germany. In 1994 and 1995, he was visiting the École Nationale Superieure des Telcommunications, Paris, France. In 2003, he was a Visiting Researcher with the Math Department, EPFL, Lausanne, Switzerland. In 2004, he was a Visiting Researcher with the Telecommunications Department, UNICAMP, Campinas, Brazil. In 2005, 2006, and 2009 he was a Visiting Researcher with the ITR, UniSA, Adelaide, Australia. In 2007, he was a Visiting Fellow with the Nokia Research Center, Helsinki, Finland. His main research interests are in lattice codes for the Gaussian and fading channels, algebraic coding theory, algebraic space-time coding, digital terrestrial television broadcasting, and digital magnetic recording.

Prof. Emanuele Viterbo is an ISI Highly Cited Researcher and Member of the Board of Governors of the IEEE Information Theory Society (2011-2013). $\mathrm{He}$ is an associate editor of the IEEE TRANSACTIONS ON INFORMATION THEORY and Guest Editor for the IEEE Journal of Selected Topics IN Signal PROCESSING Special Issue on Managing Complexity in Multiuser MIMO Systems. He Viterbo was awarded a NATO Advanced Fellowship in 1997 from the Italian National Research Council. 